\title{
Appetitive Learning Requires the Alpha1-Like Octopamine Receptor OAMB in the Drosophila Mushroom Body Neurons
}

\author{
Young-Cho Kim, Hyun-Gwan Lee, Junghwa Lim, and Kyung-An Han \\ Department of Biological Sciences, Border Biomedical Research Center Neuroscience and Metabolic Disorders Project, University of Texas at El Paso, El \\ Paso, Texas 79968
}

\begin{abstract}
Associative learning is a fundamental form of behavioral plasticity. Octopamine plays central roles in various learning types in invertebrates; however, the target receptors and underlying mechanisms are poorly understood. Drosophila provides a powerful system to uncover the mechanisms for learning and memory. Here, we report that OAMB in the mushroom body neurons mediates the octopamine's signal for appetitive olfactory learning. The octopamine receptor OAMB has two isoforms (OAMB-K3 and OAMB-AS), differing

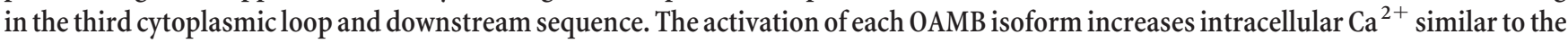
alphal adrenergic receptor, while OAMB-K3 additionally stimulates cAMP production. The oamb-null mutants showed severely impaired learning in appetitive olfactory conditioning that tests flies' capacity to learn and remember the odor associated with sugar reward. This deficit was also seen in the hypomorphic mutant with reduced OAMB expression in the mushroom bodies, the brain structure crucial for olfactory conditioning. Consistently, the oamb mutant's learning phenotype was fully rescued by conditional expression of either OAMB isoform in the mushroom body $\alpha \beta$ and $\gamma$ neurons. These results indicate that the OAMB receptor is a key molecule mediating the octopamine's signal for appetitive olfactory learning and its functional site is the mushroom body $\alpha \beta$ and $\gamma$ neurons. This study represents a critical step forward in understanding the cellular mechanism and neural circuit mediating reward learning and memory.
\end{abstract}

\section{Introduction}

Associative conditioning is vital behavioral plasticity for organisms to appropriately respond to environmental cues predicting danger or reward. In invertebrates, octopamine, the functional counterpart of mammalian norepinephrine, plays central roles in appetitive conditioning. For example, octopamine is required, in fruit flies, honeybees, and crickets, for reward-mediated olfactory learning (Farooqui et al., 2003; Schwaerzel et al., 2003; Unoki et al., 2005; Kim et al., 2007a). In the crab, octopamine also serves as an appetitive reinforcer in contextual conditioning (Kaczer and Maldonado, 2009). Like norepinephrine, octopamine's functions are exerted through particular G-protein-coupled receptors. Distinct subtypes of octopamine receptors are identified in diverse invertebrate species and have biochemical properties corresponding to alpha1, alpha2, and beta adrenergic receptors (Evans

Received June 27, 2012; revised Oct. 17, 2012; accepted Nov. 14, 2012.

Author contributions: K.-A.H. designed research; Y.-C.K., H.-G.L., and J.L. performed research;Y.-C.K. and K.-A.H. analyzed data; K.-A.H. wrote the paper.

This work was supported by National Science Foundation Grant IOS0963905 (K.-A.H.), United States Department of Agriculture Grant NIFA2010-65105-20625 (K.-A.H.), and National Institute on Minority Health and Health Disparities Grant 8G12MD007592. We thank the Bloomington stock center for providing fly lines. We also thank our undergraduate research participants, Sue Chae and Seunghee Lee, for contributing to fly collection and for image processing, respectively.

The authors declare no competing financial interests.

Correspondence should be addressed to Dr. Kyung-An Han, Department of Biological Sciences, Biosciences Building, Room 3.152, University of Texas at El Paso, 500 W. University, El Paso, TX 79968. E-mail: khan@utep.edu.

H.-G. Lee's present address: Department of Biochemistry and Molecular Biology, Pennsylvania State University, University Park, PA 16802.

DOI:10.1523/JNEUROSCI.3042-12.2013

Copyright $\odot 2013$ the authors $\quad 0270-6474 / 13 / 331672-06 \$ 15.00 / 0$ and Maqueira, 2005). Nonetheless, specific octopamine receptors mediating appetitive conditioning remain largely unknown and are of great importance to understand learning and memory mechanisms.

Drosophila melanogaster is a powerful model to uncover learning and memory mechanisms due to extensive genetic resources. The most widely adopted for this task is olfactory conditioning that tests the fly's capacity to avoid or prefer the odor associated with electric shock punishment or sugar reward for aversive or appetitive memory, respectively. Both aversive and appetitive olfactory conditioning require the mushroom body (Busto et al., 2010), a major neural structure for high-order brain functions in insects. We previously reported that D1 dopamine receptor dDA1 expressed in the mushroom body neurons mediates acquisition of aversive and appetitive memory (Kim et al., 2007b). While dumb mutants lacking dDA1 are unable to form aversive memory, they still have subnormal capacity for appetitive learning. This indicates an additional receptor or receptors crucial for appetitive learning. Several studies identify octopamine as an essential neuromodulator for appetitive olfactory conditioning in Drosophila adults and larvae. The $t \beta h$ mutants lacking octopamine are defective in appetitive olfactory conditioning while ectopic activation or blockade of octopamine neurotransmission during training induces or inhibits acquisition of appetitive memory, respectively (Schwaerzel et al., 2003; Schroll et al., 2006; Kim et al., 2007a; Honjo and Furukubo-Tokunaga, 2009). We report here that the octopamine's signal for appetitive olfactory learning is mediated by the alpha1-like octopamine receptor $\mathrm{OAMB}$ in the mushroom body neurons. 


\section{Materials and Methods}

Drosophila strains and culture. The oamb $b^{286}$, oamb ${ }^{96}$, and $o a m b^{584}$ null mutants and $o a m b^{236}$ and $o a m b^{126}$ hypomorphic alleles in the Canton-S or $r y^{506}$ genetic background (Lee et al., 2003) were used in behavioral tests along with Canton-S and $r y^{506}$ as controls. The GAL4, GAL80 $0^{t s}$, $U A S-O A M B-K 3$, and UAS-OAMB-AS lines have been previously described (Kim et al., 2007b; Lee et al., 2009). Individual transgenes were placed in the $o a m b^{286}$ or $o a m b^{96}$ background. Two independent alleles of each isoform (UAS-OAMB-K3 $3^{I}, U A S-O A M B-K 3^{I I I}, U A S-O A M B-A S^{I I}$, and $\left.U A S-O A M B-A S^{I I I}\right)$ were used for rescue experiments. Flies were reared on standard cornmeal/agar medium at $25^{\circ} \mathrm{C}$, unless otherwise stated, with $50 \%$ relative humidity and a $12 \mathrm{~h}$ light/dark cycle. The $4-$ to 7 -day-old flies of mixed sex were used for behavioral tests. In appetitive conditioning, flies were starved for $22 \mathrm{~h}$ in vials containing water-soaked Kimwipes before training.

Behavioral tests. We used the protocol described by Kim et al. (2007a) for conditioning. Briefly, flies were exposed to a first odor [conditioned stimulus (CS+)] with $2 \mathrm{~m}$ sucrose [unconditioned stimulus (US)] for appetitive conditioning or $90 \mathrm{~V}$ electric shock (US) for aversive conditioning for $1 \mathrm{~min}$. After exposure to a second odor (CS-) without sucrose or electric shock for $1 \mathrm{~min}$, flies were tested immediately or 1,3 , or $6 \mathrm{~h}$ after training in a T-maze for their preference or avoidance of CS+ odor. A second set of flies was simultaneously trained with the odors presented in a reversed order to counterbalance possible odor bias in conditioning. Performance index (PI) was calculated by subtracting the percentage of flies that made an incorrect choice from the percentage of flies that made the correct choice. An average PI of two sets of flies conditioned with counterbalanced odors was used as one data point. Odorants used for conditioning were $2 \%$ ethyl acetate (EA), and $2 \%$ isoamyl acetate (IAA). We tested control behaviors (olfactory acuity, sugar preference, and shock avoidance) as previously reported (Kim et al., 2007b). All data are reported as mean \pm SEM. For statistical analyses, we performed one-way ANOVA with post hoc Tukey-Kramer or Dunnett's tests, and analysis of covariance (ANCOVA) using Minitab 14 (Minitab, Inc.)

Immunohistochemistry. Immunostaining was conducted as previously described using mouse anti-AS (1:20) or anti-K3 antibody (1: 50 ) and Alexa 568-conjugated anti-mouse IgG (1:1000; Invitrogen) (Lee et al., 2009). Images were acquired with the LSM700 confocal microscope (Zeiss). All chemicals were purchased from Sigma and Fisher Science.

\section{Results}

\section{$o a m b$ mutants are impaired in appetitive conditioning}

Drosophila has four known octopamine receptors: OAMB and three Oct $\beta$ Rs (Han et al., 1998; Balfanz et al., 2005; Maqueira et al., 2005). Two OAMB isoforms, OAMB-K3 and OAMB-AS, produced by alternative splicing of the last exon, differ in the third cytoplasmic loop and downstream sequence. When assayed in vitro, both isoforms activate an increase in intracellular $\mathrm{Ca}^{2+}$ while OAMB-K3 also stimulates cAMP increase (Han et al., 1998; Balfanz et al., 2005). OAMB-K3 is enriched in the central complex and mushroom body crucial for associative learning and memory (Han et al., 1998), representing a good candidate to function in olfactory conditioning. When subjected to appetitive olfactory conditioning with IAA or EA as CS and $2 \mathrm{M}$ sucrose as US, $o a m b^{286}$-null mutants displayed significantly impaired performance compared with the control Canton-S flies at $3 \mathrm{~min}, 1 \mathrm{~h}$, and $3 \mathrm{~h}$ after training (Fig. $1 A$; Dunnett's test, $p<0.001, n=$ $6-12$ ). Both $o a m b^{286}$ and Canton- $S$ flies had negligible $6 \mathrm{~h}$ memory $(p=0.497)$. Notably, the $o a m b$ 's memory decay slope was not significantly different from that of Canton-S (ANCOVA: $\left.F_{(1,56)}=3.51, p=0.066\right)$, indicating that oamb mutants are defective in acquisition but not in memory retention. We examined additional null allele $o a m b^{584}$ having a different lesion (Lee et al.,
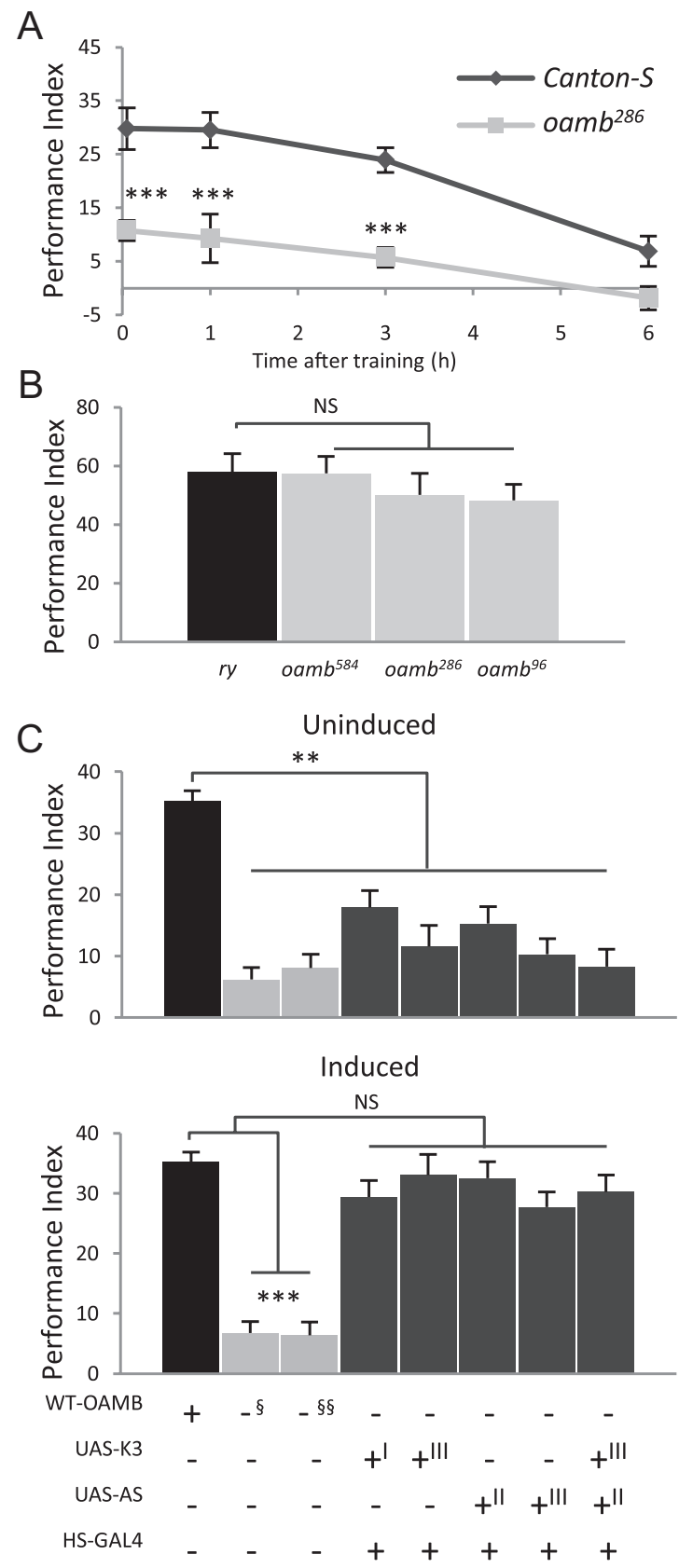

Figure 1. oamb-null mutants are impaired in appetitive learning, which is rescued by temporarily restored expression of either $0 A M B-K 3$ or $0 A M B-A S$ isoform. Flies were trained with $E A$ and IAA as CS and sucrose reward or electric shock punishment as US. A, oamb ${ }^{286}$ flies exhibited impaired performance immediately or 1 and $3 \mathrm{~h}$ after training in appetitive olfactory conditioning $\left({ }^{* * *} p<0.001, n=6-12\right.$, Dunnett's test) but their memory decay was comparable to that of the control Canon-S flies (ANCOVA, $p>0.05$ ). B, Three oamb-null alleles (oamb ${ }^{584}$, oamb ${ }^{286}$, and $0 a m b^{96}$ ) showed learning performance comparable to that in the ry control in aversive conditioning (NS, not significant; $n=6$, Tukey-Kramer test). C, The oamb $b^{286}-\left({ }^{\S}\right)$ and oamb $b^{96}$ $\left({ }^{\S \S}\right)$-null alleles and the $0 a m b^{286}$ carrying HS-GAL4 along with UAS-OAMB-KS or UAS-OAMB-AS or both, when reared at $20^{\circ} \mathrm{C}$ (Uninduced), exhibited significantly reduced performance compared with Canton-S (top). Upon heat treatment (Induced), the oamb mutants carrying the same transgenes showed learning performance comparable to that of Canton-S but significantly different from that of oamb-null alleles (bottom). Two independent UAS-OAMB lines for each isoform were used for rescue experiments and the chromosomes (| for $X, \|$ and || $\mid)$ containing the transgene are noted in the genotype table. NS, not significant; ${ }^{* *} p<0.01$; ${ }^{* * *} p<$ $0.001, n=6$, Tukey-Kramer. 
Table 1. Olfactory acuity, taste perception, and shock reactivity

\begin{tabular}{|c|c|c|c|c|c|c|c|c|c|c|}
\hline & ry & $0 a m b^{286}$ & $0 a m b^{584}$ & $0 a m b^{126}$ & $0 a m b^{96}$ & $p$ value & Canton-S & C747/UAS-AS; UAS-K3,oamb & 247/UAS-AS; UAS-K3,oamb & $p$ value \\
\hline \multicolumn{11}{|l|}{ Odor avoidance } \\
\hline $2 \%$ IAA & $58.9 \pm 5.5$ & $48.1 \pm 4.9$ & $60.9 \pm 2.3$ & $62.7 \pm 2.0$ & & 0.074 & $63.8 \pm 5.1$ & $57.6 \pm 3.4$ & $61.4 \pm 2.0$ & 0.517 \\
\hline $0.2 \%$ IAA & $31.7 \pm 5.5$ & $27.2 \pm 3.4$ & $27.9 \pm 6.2$ & $28.3 \pm 1.8$ & & 0.903 & & & & \\
\hline $2 \% \mathrm{EA}$ & $48.7 \pm 6.5$ & $45.2 \pm 4.7$ & $35.6 \pm 5.5$ & $51.6 \pm 3.0$ & & 0.169 & $65.4 \pm 1.9$ & $68.5 \pm 3.4$ & $65.9 \pm 0.7$ & 0.593 \\
\hline $0.2 \% \mathrm{EA}$ & $30.8 \pm 3.0$ & $27.7 \pm 6.4$ & $28.0 \pm 4.8$ & $33.7 \pm 1.3$ & & 0.736 & & & & \\
\hline \multicolumn{11}{|l|}{ Sugar preference } \\
\hline 2 м sucrose & $65.4 \pm 4.8$ & $60.9 \pm 2.9$ & $67.2 \pm 1.5$ & $70.1 \pm 2.7$ & & 0.150 & $70.2 \pm 3.0$ & $70.9 \pm 1.9$ & $71.4 \pm 3.2$ & 0.964 \\
\hline 0.2 m sucrose & $42.4 \pm 4.6$ & $35.6 \pm 2.8$ & $41.1 \pm 6.8$ & $41.2 \pm 3.3$ & & 0.730 & & & & \\
\hline \multicolumn{11}{|l|}{ Shock avoidance } \\
\hline $90 \mathrm{~V}$ & $56.9 \pm 3.6$ & $58.6 \pm 4.5$ & $56.3 \pm 3.6$ & & $54.2 \pm 3.7$ & 0.884 & & & & \\
\hline $30 \mathrm{~V}$ & $29.4 \pm 3.9$ & $30.3 \pm 4.1$ & $28.7 \pm 3.9$ & & $30.7 \pm 4.7$ & 0.987 & & & & \\
\hline
\end{tabular}

The control, oamb, and rescue flies showed comparable avoidance of the CS odors and electric shock presented at two different concentrations and intensities, respectively, and similar preference for 0.2 or $2 \mathrm{~m} \mathrm{sucrose}(n=6-12)$.

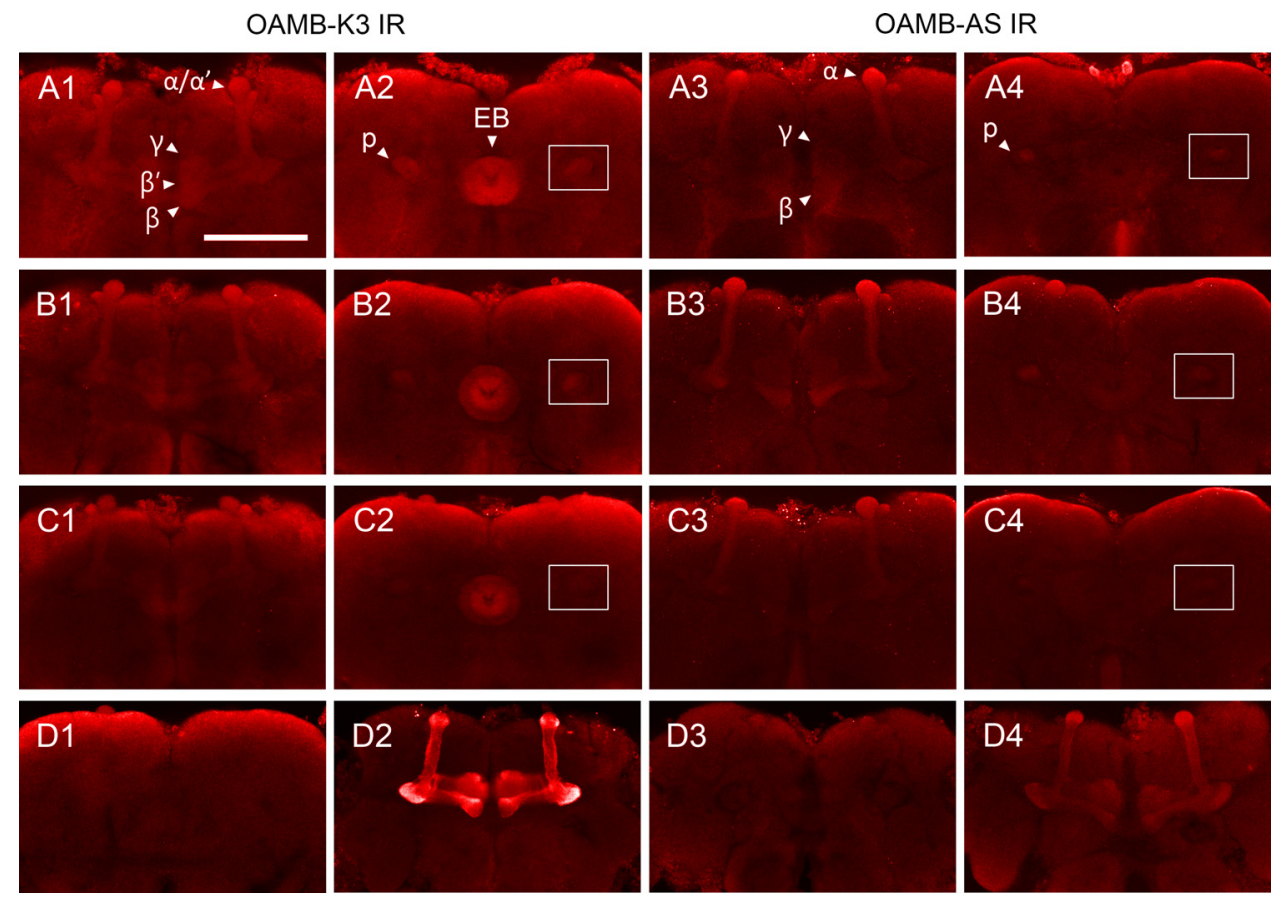

Figure 2. OAMB expression. A1-D4, The whole-mount brains of Canton-S (A1-A4), oamb ${ }^{236}\left(\right.$ B1-B4), oamb ${ }^{126}\left(\right.$ C1-C4), oamb $b^{286}$ (D1) and the oamb ${ }^{286}$ with MB247-GAL4/UAS-0AMB-K3 $(\mathbf{D 2}, \mathbf{D 3})$ or MB247-GAL4/UAS-OAMB-AS (D4) were stained with anti-K3 (two right columns) or anti-AS (two left columns) antibody. The optical sections at the level of the mushroom body lobes (the first and third columns) and pedunculi and the ellipsoid body (the second and forth columns) are shown. In Canton-S and oamb ${ }^{236}, 0 \mathrm{AMB}-\mathrm{AS}$ is visible in the $\alpha \beta$ and $\gamma$ lobes and pedunculi, but not in the ellipsoid body, whereas OAMB-K3 is visible in the ellipsoid body as well. Both OAMB-K3 and -AS levels are significantly reduced in the oamb ${ }^{126}$ mushroom body neuropil (C1-C4) and absent in oamb ${ }^{286}(\mathbf{D 1}, \mathbf{D 3})$. The pedunculus area is boxed for comparison. OAMB expression induced by MB247-GAL 4 is visible in the mushroom body $\alpha \beta$ and $\gamma$ lobes in the isoform-specific manner (D2-D4). For example, OAMB in MB247-GAL4/UAS-OAMB-AS;0amb ${ }^{286}$ (D4), but not in UAS-0AMB-K3/+;MB247-GAL4/+;0amb ${ }^{286}$ (D3), is detectable with anti-AS antibody. IR, Immunoreactivity; EB, ellipsoid body; p, pedunculus. All images are at the same magnification. Scale bar, $100 \mu \mathrm{m}$.

2003) and observed the same phenotype as oamb ${ }^{286}$ (see Fig. 3A). The oamb's poor performance was not due to defects in sensorimotor behaviors because oamb mutant and control flies exhibited comparable avoidance of the CS odors and preference for sucrose presented at the concentrations used during training or the concentrations diluted 10 times (Table 1). Therefore, impaired learning in oamb mutants is likely due to their inability to associate CS + with US. To explore the specificity of oamb's deficit, we examined aversive olfactory conditioning. When trained with electric shock as US and the same odors as CS, oamb mutants showed normal performance right after training (Fig. $1 B$; ANOVA, $\left.F_{(3,20)}=0.64, p=0.599, n=6\right)$ or $1 \mathrm{~h}$ after training $(r y$ $\left.46.6 \pm 5.98 ; \mathrm{oamb}^{286}, 54.5 \pm 0.94 ; p=0.219, n=6\right)$. These results indicate that $o a m b$ mutants are impaired in appetitive, but not aversive, olfactory learning.
Either OAMB isoform is sufficient to reinstate normal appetitive learning

OAMB is expressed in the nervous system during development (data not shown). We asked whether the oamb's phenotype is due to physiological absence of OAMB or developmental anomaly. In this study, we also addressed functional contribution of two $\mathrm{OAMB}$ isoforms having distinct downstream signaling capacity to appetitive learning. For this task, we used HS-GAL4 (GAL4 expression under heat-inducible $h s p 70$ promoter) to restore OAMB expression in oamb mutants at the adult stage (Lee et al., 2009). The transgenic oamb mutants carrying HS-GAL4 along with UAS-OAMB-K3 or UAS-OAMB-AS as well as Canton-S and $o a m b$ mutants were treated with or without heat shock at $37^{\circ} \mathrm{C}$ for $1 \mathrm{~h}$ and subjected to appetitive conditioning next day. To eliminate potential effects of transgene insertion sites, we used two 
independent UAS lines carrying the transgene inserted in different chromosomes for each isoform. In the absence of heat treatment (i.e., no induction), the oamb mutants carrying both HS-GAL4 and UAS-OAMB showed poor learning similar to oamb mutants (Fig. $1 C$, top; ANOVA, $F_{(7,40)}=12.04, p<0.0001$, $n=6)$. However, the oamb mutants with heat-induced OAMB expression, either isoform alone or both isoforms together, had learning scores comparable to that of Canton-S (Fig. 1C, bottom; ANOVA: $\left.F_{(7,40)}=20.87, p<0.0001 ; n=6\right)$. These results indicate that the oamb's phenotype is not due to abnormal development but due to the absence of OAMB, presumably at the time of learning. Also, either OAMB isoform is sufficient to reinstate normal learning in $o a m b$ mutants.

\section{The OAMB's functional site for appetitive learning is the mushroom body}

OAMB-K3 is enriched in the mushroom body $\alpha \beta, \alpha^{\prime} \beta^{\prime}$, and $\gamma$ lobes, pedunculi, and ellipsoid body (Fig. 2A1,A2) (Han et al., 1998). To identify the expression pattern of OAMB-AS, we conducted immunohistochemical analysis using the AS-specific antibody (Lee et al., 2009). Overall, OAMB-AS immunoreactivity was visible in the mushroom body $\alpha \beta$ and $\gamma$ lobes and pedunculi, but not evident in the $\alpha^{\prime} \beta^{\prime}$ lobes or ellipsoid body in the wildtype Canton-S (Fig. 2A3,A4). Since the oamb's phenotype is rescued by either isoform, we hypothesized that the $\alpha \beta$ and $\gamma$ lobes where both isoforms are commonly expressed may serve as a neural site for OAMB's function in appetitive learning. Consistent with the notion, oamb hypomorphic alleles with different genetic lesions (Lee et al., 2003) have distinctive OAMB expression patterns and learning capacities. When stained with the isoform specific antibodies, $o a m b^{126}$ showed significantly reduced OAMB-K3 and OAMB-AS in the mushroom body neuropil, but normal OAMB-K3 in the ellipsoid body (Fig. 2C1-C4). oamb ${ }^{236}$, on the contrary, had normal OAMB-K3 and OAMB-AS expression patterns (Fig. 2B1-B4). In appetitive olfactory conditioning, $o a m b^{126}$, but not $o a m b^{236}$, exhibited poor performance similar to oamb-null mutants (Fig. 3A; ANOVA: $F_{(4,53)}=7.82, p<0.0001$, $n=6-18)$. These observations further support the theory that $\mathrm{OAMB}$ in the mushroom body neurons mediates appetitive olfactory learning.

To test whether the mushroom body OAMB is necessary for appetitive learning and sufficient to reinstate the oamb's phenotype, we used the mushroom body drivers C747-GAL4 expressed in all three lobe neurons and MB247-GAL4 expressed in the $\alpha \beta$ and $\gamma$ lobe neurons (Kim et al., 2007b). When MB247-GAL4 was used with UAS-OAMB-K3 or UAS-OAMB-AS in the $o a m b^{286}$ null mutant, respective OAMB immunoreactivity was conspicuously visible in the mushroom body $\alpha \beta$ and $\gamma$ lobes (Fig. $2 D 2, D 4)$ but not in other neurons, including the ellipsoid body (data not shown). OAMB expression induced by either C747GAL4 or MB247-GAL4 during development and adulthood fully rescued the $o a m b^{286}$ mutant's learning phenotype (Fig. $3 B$; ANOVA: $\left.F_{(3,37)}=27.18, p<0.0001, n=6-17\right)$. For temporally restricted OAMB expression, we added temperature-sensitive GAL $80^{\text {ts }}$. GAL80 ${ }^{\text {ts }}$ binds to GAL4 to sequester it from activating UAS at $22^{\circ} \mathrm{C}$ but can no longer bind to GAL4 at $30^{\circ} \mathrm{C}$, allowing it to activate UAS. To induce OAMB expression in the adult mushroom body neurons, the oamb ${ }^{286}$ mutants carrying tubPGAL80 ${ }^{\text {ts }}$, MB247-GAL4, and UAS-OAMB (-K3 or -AS or both) were reared at $30^{\circ} \mathrm{C}$ for $3 \mathrm{~d}$ and subjected to conditioning. Similar to the HS-GAL4 experiment, $o a m b^{286}$ with restored expression of OAMB-K3, OAMB-AS, or both in the adult mushroom body $\alpha \beta$ and $\gamma$ neurons had learning scores similar to that of Canton-S
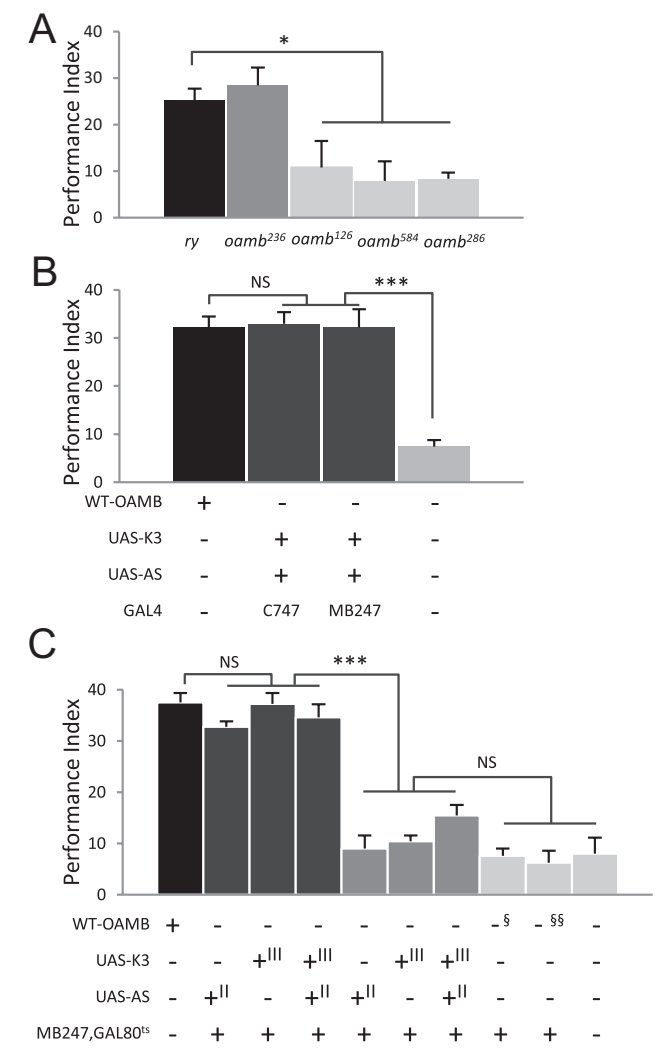

Figure 3. The OAMB's functional site for appetitive learning is the mushroom body $\alpha \beta$ and $\gamma$ neurons. $A$, Behavioral analysis of hypomorphic oamb alleles. oamb $b^{236}$ with normal mushroom body $0 A M B$ expression displayed performance comparable to that of the ry control; however, oamb ${ }^{126}$ with significantly reduced mushroom body OAMB showed severe deficit in acquisition of appetitive memory like oamb $b^{286}$ - and oamb $b^{584}$-null mutants ( $n=6-18$ ). $\boldsymbol{B}$, Transgenic OAMB expression induced by the mushroom body driver C747-GAL4 or MB247-GAL4 during development and adulthood fully rescued the oamb's learning phenotype ( $n=6-17)$. C, Normal appetitive learning in oamb mutants was reinstated by temporarily restored OAMB-K3 or OAMB-AS or both in the adult mushroom body $\alpha \beta$ and $\gamma$ neurons. The oamb mutants carrying MB247-GAL4 and tubP-GAL80 ${ }^{\text {ts }}$ along with UAS-OAMB-K3 or UAS-OAMB-AS or both, upon mild heat treatment before training (dark gray columns), showed learning performance comparable to that of Canton- $S$ ( $n=6$ for each genotype). The same genotypes without heat treatment (mid-gray columns) or the $0 a m b^{286}\left({ }^{\S}\right)$ and $0 a m b^{286} / 0 a m b^{96}\left({ }^{\S \S}\right)$ carrying only MB247-GAL4 and tub-GAL80 ${ }^{\text {ts }}$ subjected to the same temperature shift (light gray columns), however, showed poor learning similar to oamb ${ }^{286}$. II and III in the genotype table denote the chromosomes containing the transgene. NS, Not significant; ${ }^{*} p<0.05 ;{ }^{* * *} p<$ 0.001, Tukey-Kramer.

(Fig. 3C; ANOVA, $F_{(9,50)}=40.02, p<0.0001, n=6$ ). The $\mathrm{oamb}^{286}$ mutants carrying MB247-GAL4, GAL80 ${ }^{\text {ts }}$, and UAS$\mathrm{OAMB}$ kept at $22^{\circ} \mathrm{C}$ (i.e., no induction), however, had low learning scores comparable to that of $o a m b^{286}$. The oamb ${ }^{286}$ mutants carrying MB247-GAL4 and GAL80 ${ }^{\text {ts }}$ subjected to the same temperature shift showed impaired learning as well. This indicates that the reinstated learning is not contributed by the transgenes MB247-GAL4 and GAL80 ${ }^{\text {ts }}$ or temperature shift. Together, these observations clarify the mushroom body $\alpha \beta$ and $\gamma$ neurons as the major neural site for the physiological OAMB's function in appetitive olfactory learning.

\section{Discussion}

Octopamine is a major neuromodulator mediating appetitive US information in olfactory conditioning. The receptors conveying the octopamine's signal to initiate the learning cascade are critical but unknown. In this report, we demonstrate that the alpha1-like octopamine receptor OAMB is essential for appetitive olfactory 


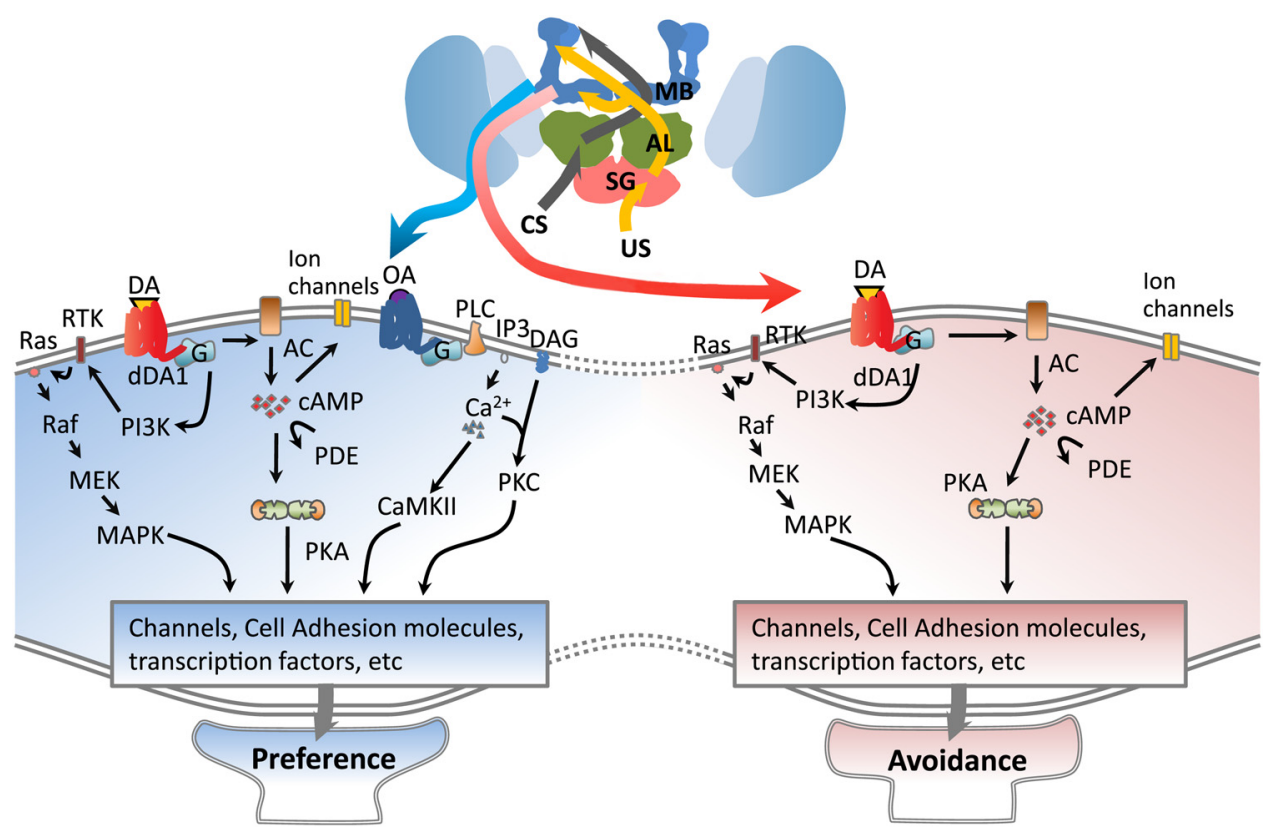

Figure 4. Working model for appetitive and aversive learning mediated by OAMB and dDA1 in the mushroom body. Top, Schematic fly brain. The mushroom body (MB) calyx colored light blue receives (S information from the antennal lobe (AL; gray pathway). Sugar input (appetitive US) received in the tarsi and proboscis is delivered to the mushroom body lobes via octopamine (OA) and dopamine (DA) neurons (yellow pathway). Similarly, electric shock input (aversive US) received in the legs is delivered to the mushroom body lobes (dark blue) via dopamine neurons. When activated by dopamine conveying aversive US input, dDA1 in the mushroom body lobes may trigger the signal cascade involving CAMP and MAP kinases to modify mushroom body neuronal output for avoidance behavior (red pathway). In contrast, dopamine and octopamine conveying appetitive US input bind to dDA1 and OAMB, respectively, to activate the appetitive learning cascade involving multiple effectors such as CAMP, MAPK, CaMKII and/or PKC, some of which may interact with each other (data not shown for simplicity). Overall, the appetitive-learning cascade activated by OAMB and dDA1 together (blue-shaded area) may be distinct from the aversive-learning cascade activated by dDA1 alone (red-shaded area), and alter mushroom body output favoring preference behavior. AC, Adenylyl cyclase; CaMKII, calcium/calmodulin-dependent protein kinase Il; DAG, diacylglycerol; PDE, phosphodiesterase; G, heterotrimeric G protein complex; MAPK, MAP kinase; MEK, MAP kinase kinase; PI3K, PI3 kinase; PLC, phospholipase C; PKA, protein kinase A; PKC, protein kinase C; RTK, receptor tyrosine kinase; SG, subesophageal ganglion.

learning and its functional site is the mushroom body neurons. The OAMB's role in this behavioral plasticity is physiological, rather than developmental. This is consistent with the observation that octopamine feeding before training rescues the $t \beta h \mathrm{mu}-$ tant's learning phenotype (Schwaerzel et al., 2003). Also in Drosophila larvae, octopamine neuronal output is required during training and ectopic activation of octopamine neurons replaces sugar US reinforcement in olfactory conditioning (Schroll et al., 2006; Honjo and Furukubo-Tokunaga, 2009). These observations indicate OAMB as a key receptor conveying sugar US reinforcement in the mushroom body neurons for acquisition of appetitive memory.

The mushroom body is known to be crucial for appetitive olfactory learning and memory. The mushroom body ablation abolishes appetitive odor learning in blowflies (Ikeda et al., 2005), while octopamine injection into the calyx area substitutes sugar reinforcement in associative proboscis extension reflex (Hammer and Menzel, 1998). In Drosophila olfactory conditioning, distinct mushroom body neurons are involved in appetitive memory consolidation and retrieval. For example, output of the $\gamma$ and $\alpha \beta$ neurons is important for retrieval of short- and longterm memory of sugar reward, respectively (Trannoy et al., 2011), and the $\alpha^{\prime} \beta^{\prime}$ neurons for consolidation (Krashes and Waddell, 2008). The expression patterns of OAMB-AS and MB247-GAL4 indicate acquisition of sugar memory involving OAMB in the $\alpha \beta$ and $\gamma$, but not $\alpha^{\prime} \beta^{\prime}$, neurons. Octopamine immunoreactivity is observed in the calyx and $\gamma$ lobes (Sinakevitch and Strausfeld, 2006). While the $\alpha \beta$ and $\alpha^{\prime} \beta^{\prime}$ lobes are devoid of octopamine immunoreactivity, MARCM and GRASP analyses that allow high spatial resolution imaging reveal the octopamine neurons innervating the $\alpha \beta$ lobes (Zhou et al., 2012).
The calyx has OAMB-AS, but not OAMB-K3, immunoreactivity and this immunoreactivity is contributed by extrinsic, but not intrinsic, mushroom body neurons (J. Lim and K.-A. Han, unpublished observations). Therefore, the octopamine neurons innervating the $\alpha \beta$ and $\gamma$ lobes likely deliver sugar US information to OAMB for acquisition of reward memory. Appetitive shortand long-term memories are shown to be formed in parallel in $\gamma$ and $\alpha \beta$ mushroom lobe neurons, respectively (Trannoy et al., 2011). Consistently, OAMB in the $\gamma$ and $\alpha \beta$ lobes may function independently for acquisition of appetitive odor memories.

We previously demonstrated that the MB247-positive $\alpha \beta$ and $\gamma$ neurons also require D1 dopamine receptor dDA1 for appetitive as well as aversive learning (Kim et al., 2007b). While dumb mutants lacking dDA1 are completely impaired in aversive learning, they show reduced yet detectable acquisition of appetitive memory. OAMB is not required for aversive learning. Thus, in the MB247-positive mushroom body neurons, acquisition of appetitive and aversive memory is distinguished by recruitment of both $\mathrm{OAMB}$ and $\mathrm{dDA} 1$, or $\mathrm{dDA} 1$ alone, respectively. A recent study (Qin et al., 2012) clarifies that dDA1 in the $\gamma$ neurons is sufficient for aversive learning and memory. It is conceivable that appetitive learning involves $\mathrm{OAMB}$ and $\mathrm{dDA} 1$ in the $\gamma$ neurons as well. Regardless of whether OAMB and dDA1 act in the same or different neuropil, appetitive and aversive learning may be differentiated by intracellular signals and neuronal output induced by $\mathrm{OAMB}$ and $\mathrm{dDA} 1$ together or $\mathrm{dDA} 1$ alone. As noted, two OAMB isoforms have distinct downstream signals. OAMB-K3, but not -AS, stimulates cAMP generation, whereas both isoforms increase intracellular $\mathrm{Ca}^{2+}$ levels. The capacity of either OAMB isoform to rescue the oamb's learning phenotype points to intracellular $\mathrm{Ca}^{2+}$, rather than cAMP, as a downstream effector. 
dDA1, however, activates the cAMP pathway. Therefore, the learning cascade activated by $\mathrm{Ca}^{2+}$ (via octopamine/OAMB) and cAMP (via dopamine/dDA1) may induce mushroom body output, triggering preference behavior, whereas the cascade involving downstream effectors of cAMP alone (via dopamine/dDA1) causes avoidance behavior (Fig. 4). This model is consistent with the findings that the mushroom body RUTAGAGA-adenylyl cyclase and protein kinase A are important for both appetitive and aversive conditioning (Gervasi et al., 2010; Trannoy et al., 2011). $\mathrm{G}(\mathrm{o})$ activation is also required for both appetitive and aversive learning (Madalan et al., 2012), and possibly works downstream of dDA1. The effectors critical only for appetitive learning are unknown at present. The findings reported here provide a key framework to elucidate the appetitive learning cascade and neural circuit.

Alternative splicing is a common mechanism for increasing protein diversity in eukaryotes and occurs in 95\% of the human multiexon genes (Pan et al., 2008). Alphala adrenergic receptor, a human homolog of OAMB, has multiple isoforms generated by alternative splicing as well, but expression patterns and functional significance of individual isoforms in vivo are unknown. Distinct expression patterns of two OAMB isoforms in the brain that we report here are novel. Of particular interest is the presence of OAMB-K3, but not -AS, in the ellipsoid body known to be important for long-term memory formation in aversive olfactory conditioning and visual place learning (Wu et al., 2007; Ofstad et al., 2011). It is tempting to speculate that OAMB may have an isoform-specific function in the ellipsoid body for olfactory memory consolidation or visual memory acquisition.

\section{References}

Balfanz S, Strünker T, Frings S, Baumann A (2005) A family of octopamine [corrected] receptors that specifically induce cyclic AMP production or $\mathrm{Ca} 2+$ release in Drosophila melanogaster. J Neurochem 93:440-451. CrossRef Medline

Busto GU, Cervantes-Sandoval I, Davis RL (2010) Olfactory learning in Drosophila. Physiology 25:338-346. CrossRef Medline

Evans PD, Maqueira B (2005) Insect octopamine receptors: a new classification scheme based on studies of cloned Drosophila G-protein coupled receptors. Invert Neurosci 5:111-118. CrossRef Medline

Farooqui T, Robinson K, Vaessin H, Smith BH (2003) Modulation of early olfactory processing by an octopaminergic reinforcement pathway in the honeybee. J Neurosci 23:5370-5380. Medline

Gervasi N, Tchénio P, Preat T (2010) PKA dynamics in a Drosophila learning center: coincidence detection by rutabaga adenylyl cyclase and spatial regulation by dunce phosphodiesterase. Neuron 65:516-529. CrossRef Medline

Hammer M, Menzel R (1998) Multiple sites of associative odor learning as revealed by local brain microinjections of octopamine in honeybees. Learn Mem 5:146-156. Medline

Han KA, Millar NS, Davis RL (1998) A novel octopamine receptor with preferential expression in Drosophila mushroom bodies. J Neurosci 18: 3650-3658. Medline

Honjo K, Furukubo-Tokunaga K (2009) Distinctive neuronal networks and biochemical pathways for appetitive and aversive memory in Drosophila larvae. J Neurosci 29:852-862. CrossRef Medline

Ikeda K, Numata H, Shiga S (2005) Roles of the mushroom bodies in olfac- tory learning and photoperiodism in the blow fly Protophormia terraenovae. J Insect Physiol 51:669-680. CrossRef Medline

Kaczer L, Maldonado H (2009) Contrasting role of octopamine in appetitive and aversive learning in the crab Chasmagnathus. PLoS One 4:e6223. CrossRef Medline

Kim YC, Lee HG, Han KA (2007a) Classical reward conditioning in Drosophila melanogaster. Genes Brain Behav 6:201-207. CrossRef Medline

Kim YC, Lee HG, Han KA (2007b) D1 dopamine receptor dDA1 is required in the mushroom body neurons for aversive and appetitive learning in Drosophila. J Neurosci 27:7640-7647. CrossRef Medline

Krashes MJ, Waddell S (2008) Rapid consolidation to a radish and protein synthesis-dependent long-term memory after single-session appetitive olfactory conditioning in Drosophila. J Neurosci 28:3103-3113. CrossRef Medline

Lee HG, Seong CS, Kim YC, Davis RL, Han KA (2003) Octopamine receptor OAMB is required for ovulation in Drosophila melanogaster. Dev Biol 264:179-190. CrossRef Medline

Lee HG, Rohila S, Han KA (2009) The octopamine receptor OAMB mediates ovulation via $\mathrm{Ca} 2+/$ calmodulin-dependent protein kinase II in the Drosophila oviduct epithelium. PLoS One 4:e4716. CrossRef Medline

Madalan A, Yang X, Ferris J, Zhang S, Roman G (2012) G(o) activation is required for both appetitive and aversive memory acquisition in Drosophila. Learn Mem 19:26-34. CrossRef Medline

Maqueira B, Chatwin H, Evans PD (2005) Identification and characterization of a novel family of Drosophila beta-adrenergic-like octopamine G-protein coupled receptors. J Neurochem 94:547-560. CrossRef Medline

Ofstad TA, Zuker CS, Reiser MB (2011) Visual place learning in Drosophila melanogaster. Nature 474:204-207. CrossRef Medline

Pan Q, Shai O, Lee LJ, Frey BJ, Blencowe BJ (2008) Deep surveying of alternative splicing complexity in the human transcriptome by highthroughput sequencing. Nat Genet 40:1413-1415. CrossRef Medline

Qin H, Cressy M, Li W, Coravos JS, Izzi SA, Dubnau J (2012) Gamma neurons mediate dopaminergic input during aversive olfactory memory formation in Drosophila. Curr Biol 22:608-614. CrossRef Medline

Schroll C, Riemensperger T, Bucher D, Ehmer J, Völler T, Erbguth K, Gerber B, Hendel T, Nagel G, Buchner E, Fiala A (2006) Light-induced activation of distinct modulatory neurons triggers appetitive or aversive learning in Drosophila larvae. Curr Biol 16:1741-1747. CrossRef Medline

Schwaerzel M, Monastirioti M, Scholz H, Friggi-Grelin F, Birman S, Heisenberg M (2003) Dopamine and octopamine differentiate between aversive and appetitive olfactory memories in Drosophila. J Neurosci 23:10495-10502. Medline

Sinakevitch I, Strausfeld NJ (2006) Comparison of octopamine-like immunoreactivity in the brains of the fruit fly and blow fly. J Comp Neurol 494:460 - 475. CrossRef Medline

Trannoy S, Redt-Clouet C, Dura JM, Preat T (2011) Parallel processing of appetitive short- and long-term memories in Drosophila. Curr Biol 21: 1647-1653. CrossRef Medline

Unoki S, Matsumoto Y, Mizunami M (2005) Participation of octopaminergic reward system and dopaminergic punishment system in insect olfactory learning revealed by pharmacological study. Eur J Neurosci 22:1409-1416. CrossRef Medline

Wu CL, Xia S, Fu TF, Wang H, Chen YH, Leong D, Chiang AS, Tully T (2007) Specific requirement of NMDA receptors for long-term memory consolidation in Drosophila ellipsoid body. Nat Neurosci 10:1578-1586. CrossRef Medline

Zhou C, Huang H, Kim SM, Lin H, Meng X, Han KA, Chiang AS, Wang JW, Jiao R, Rao Y (2012) Molecular genetic analysis of sexual rejection: roles of octopamine and its receptor OAMB in Drosophila courtship conditioning. J Neurosci 32:14281-14287. CrossRef Medline 